\title{
Mantle cell lymphoma complicated by multiple widespread extranodal gastrointestinal lesions
}

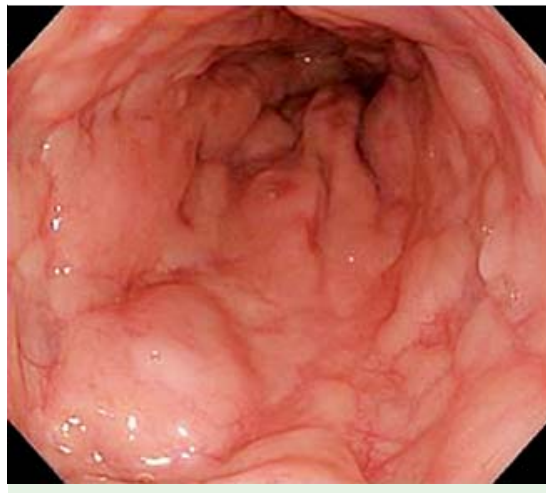

Fig. 1 Upper gastrointestinal endoscopy in a 65 -year-old man hospitalized with generalized lymphadenopathy showed multiple flat tuberous lesions in the middle to lower esophagus, diagnosed as multiple lymphomatous polyposis (MLP). Excavated lesions were also present on the apex of the esophagus.

A 65-year-old man was hospitalized in our department with generalized lymphadenopathy. He was diagnosed with mantle cell lymphoma (MCL) complicated by peripheral tumorigenesis and marrow infiltration in a biopsy of cervical lymph nodes. Upper gastrointestinal endoscopy showed multiple tuberous lesions in the lower esophagus ( $\bullet$ Fig. 1 ) and the great curvature of the stomach ( $\bullet$ Fig. 2 a). These findings were diagnosed as multiple lymphomatous polyposis (MLP). Narrow-band imaging (NBI) with magnification showed microscopically dendritic abnormal vascular hyperplasia on tuberous parts of intragastric MLP lesions ( $\bullet$ Fig. 2b). Lower gastrointestinal endoscopy showed a tuberous lesion in the cecum ( $\bullet$ Fig.3 a ) and multiple small polypoid lesions throughout the large intestine ( $\bullet$ Fig. $\mathbf{3 b}$ ). Immunohistologic staining of biopsy tissues from the stomach ( $\bullet$ Fig.4a-d) and large intestine ( Fig.5a-d) gave positive results for CD5, CD20, and cyclin D1 colocalized with nuclear staining. These findings permitted a definite diagnosis of gastrointestinal lesions of MCL. Treatment is planned with R-hyper CVAD/MA followed by high dose chemotherapy combined with autologous peripheral blood stem cell transplantation.
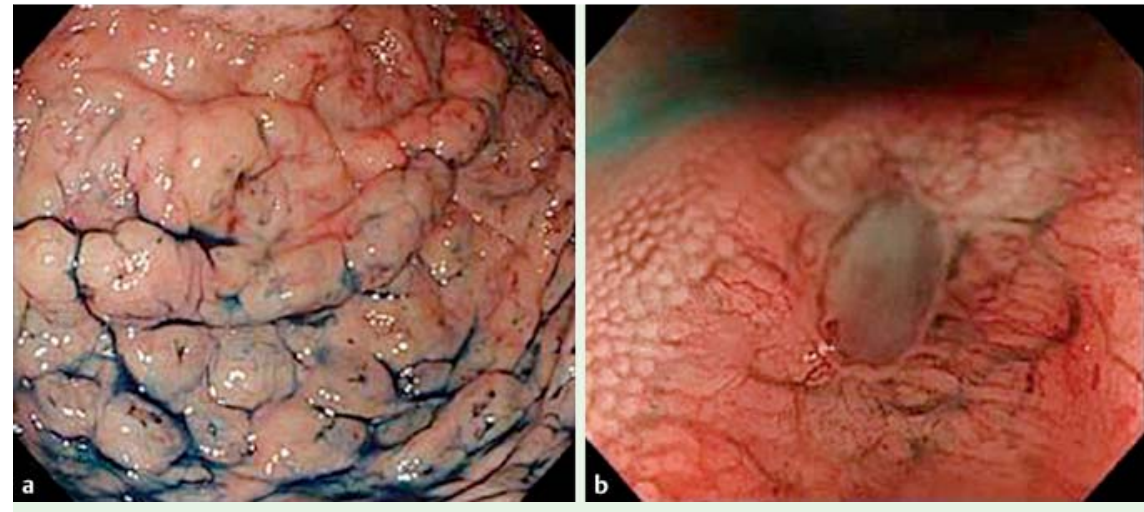

Fig.2 a Multiple flat tuberous lesions in the middle great curvature to lower curvature of the stomach, and excavated lesions on the apex of the stomach, were diagnosed as multiple lymphomatous polyposis (MLP), similarly to the lesions in the esophagus. b Narrow-band imaging (NBI) with magnification showed microscopically dendritic abnormal vascular hyperplasia on the tuberous parts of intragastric MLP lesions.

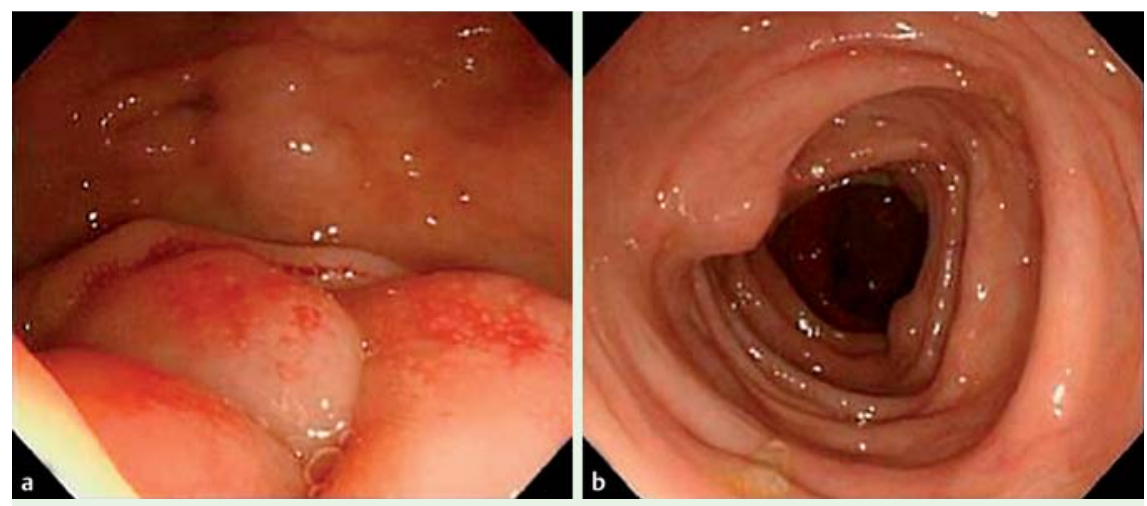

Fig. 3 Lower gastrointestinal endoscopy showed: a lesions resembling submucosal tumors with few epithelial changes in the cecum; $\mathbf{b}$ multiple small polypoid lesions present throughout the large intestine.

MCL is a B-cell lymphoma that accounts for $5 \%-10 \%$ of cases of malignant lymphoma, and $75 \%$ of cases of MCL at diagnosis are at an advanced stage (III or IV) [1]. Extranodal infiltration is found most frequently in the gastrointestinal tract, but $5.7 \%$ of cases show extranodal infiltration in esophageal lesions [2]. There are a few reports of narrow-band imaging with magnifying endoscopy for gastrointestinal lesions of MCL, and this technique has been described in a few cases of dendritic abnormal vascular hyperplasia [3]. High dose chemotherapy combined with autologous peripheral blood stem cell trans- plantation after R-hyper CVAD/MA treatment is effective for advanced MCL [4]. A combination of rituximab and bendamustine as intensive chemotherapy may also be useful for cases that are difficult to treat [5].

Endoscopy_UCTN_Code_CCL_1AB_2AD

Competing interests: None 


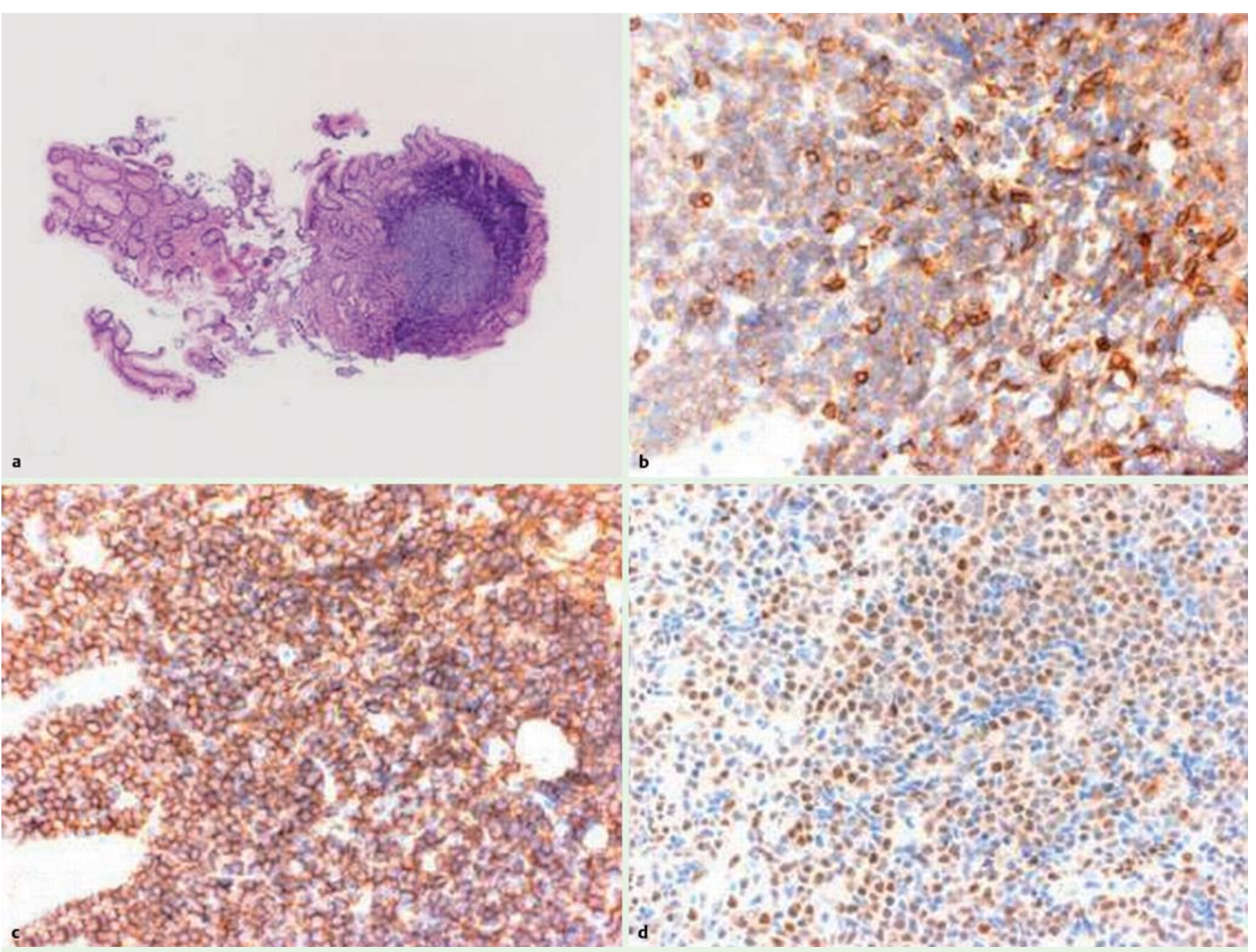

Fig. 4 Staining of biopsy tissues from lesions in the stomach revealed dense infiltration and proliferation of small to medium sized lymphocytes. Immunohistologic staining was positive for CD5, CD20, and cyclin D1 in nuclei. These findings led to a diagnosis of mantle cell lymphoma (MCL). a Hematoxylin and eosin (× 10); b CD5 (× 100); c CD20 (×100); d cyclin D1 (× 100).

\section{Tomoya lida, Suguru Nakagaki, Shuji Satoh, Haruo Shimizu, Hiroyuki Kaneto}

Department of Gastroenterology, Muroran City General Hospital, Muroran, Hokkaido, Japan

\section{References}

1 Zhou $Y$, Wang $H$, Fang $W$ et al. Incidence trends of mantle cell lymphoma in the United States between 1992 and 2004. Cancer 2008; 113: $791-798$

2 Iwamuro M, Okada $H$, Kawahara $Y$ et al. Endoscopic features and prognosis of mantle cell lymphoma with gastrointestinal involvement. World J Gastroenterol 2010; 16 : $4661-4669$
3 Norimura $D$, Isomoto $H$, Tsukasaki $K$ et al. Gastric involvement by mantle cell lymphoma observed by magnified endoscopy with narrow-band imaging. Gastrointest Endosc 2012; 75: 421-422

4 Ritchie DS, Seymour JF, Grigg AP et al. The hyper-CVAD-rituximab chemotherapy programme followed by high-dose busulfan, melphalan and autologous stem cell transplantation produces excellent event-free survival in patients with previously untreated mantle cell lymphoma. Ann Hematol 2007; 86: $101-105$

5 Rummel MJ, Niederle N, Maschmeyer G et al. Bendamustine plus rituximab versus $\mathrm{CHOP}$ plus rituximab as first-line treatment for patients with indolent and mantle-cell lymphoma: an open-label, multicenter, randomised, phase 3 non-inferiority trial. Lancet 2013; 381: $1203-1210$
Bibliography

DOI http://dx.doi.org/

10.1055/s-0034-1377762

Endoscopy 2015; 47: E91-E93

(C) Georg Thieme Verlag KG

Stuttgart · New York

ISSN 0013-726X

\section{Corresponding author \\ Tomoya lida, MD}

Department of Gastroenterology

Muroran City General Hospital

8-1 3-chome

Yamate-cho

Muroran

Hokkaido

051-8512

Japan

Fax: +81-143-226867

tomoya.iida.0306@gmail.com 


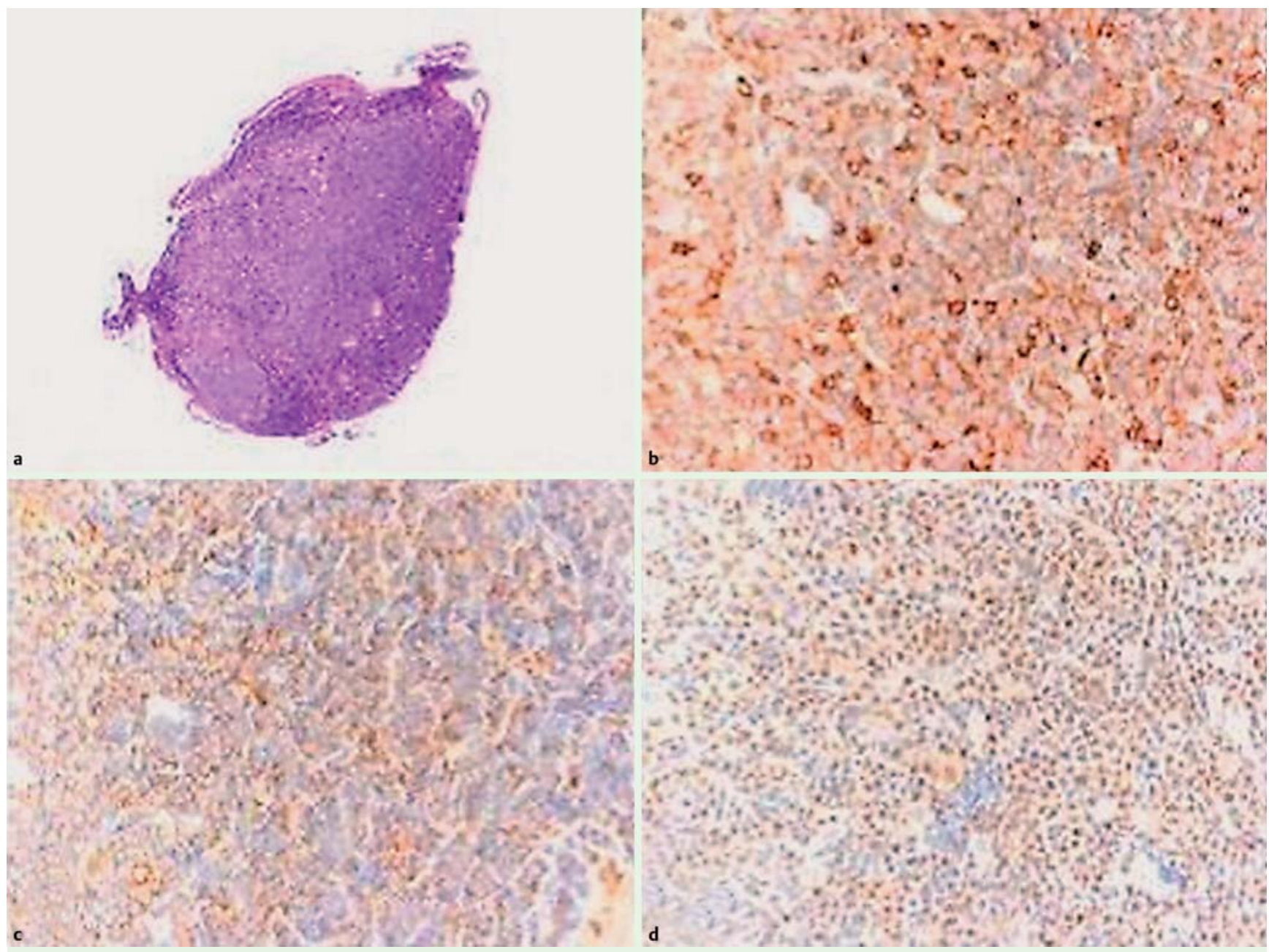

Fig. 5 Staining of biopsy tissues from lesions in the large intestine resulted in a diagnosis of mantle cell lymphoma (MCL), similarly to the diagnosis of lesions in the stomach. a Hematoxylin and eosin (×10); b CD5 (× 100); c CD20 (×100); d cyclin D1 (×100). 